\title{
Using SIMULINK® to model and simulate supplier evaluation and selection problem
}

\author{
$\underline{\text { Adnan M. Abu-Ajamieh }}{ }^{\mathrm{a}}$, Lee Luong ${ }^{\mathrm{a}}$, Romeo Marian ${ }^{\mathrm{a}}$ \\ ${ }^{a}$ University of South Australia, School of Engineering, Mawson Lakes Boulevard Mawson Lakes, South \\ Australia 5095, Australia
}

Email: Adnan.Abu-Ajamieh@mymail.unisa.edu.au

\begin{abstract}
This paper presents a new conceptual model for supplier evaluation and selection decision support system using simulation. The model is based on the concept of Supplier Product-Profile Equivalent Cost (SPPEC) introduced previously by the authors. This paper intends to develop the previous model using simulation to generate a large number of suppliers' profiles that will enable a better and more accurate decision of best supplier based on a range of performance indicators.
\end{abstract}

Simulation is used to generate multiple values of the suppliers' performances summarised as an Actual SupplierProduct Profile (ASPP). In the process, each supplier has a range of different types of selection criteria (decision variables) that will change representing the supplier performance variations according to the process/product characteristics hence generating different numbers of scenarios of supplier-product performances which will then be converted into an equivalent cost named Supplier Product Profile Equivalent Cost (SPPEC) simplifying the task for the decision maker by selecting the supplier that generates the minimum cost; a simple, yet very powerful tool to use.

This methodology will produce a more realistic decision and will increase the confidence level in the decision making process. The basic simulation model is explained using an illustrative case where MATLAB® \& SIMULINK® were used to build and simulate the model because of their strong computational capabilities and the availability of a wide range of tool boxes that can be easily and seamlessly integrated in the model.

The decision model can accommodate any number of criteria, any number of potential suppliers and any type of input data (qualitative or quantitative).

In addition to its simplicity, the interpretation of the results is straightforward. The use of equivalent cost to select the best supplier makes it more practical and easier to understand by any person in any industry.

It is also important to state that the model is a very flexible one. It can be tailored to suit any industry and to deal with any type of data. This may reduce the need to establish complicated and expensive data collection systems.

Another important advantage of this model comes from the wide range of potential applications; as it is very easy to utilise the concept in modelling and solving any multi-alternative decision problem. In further work, using Neural Network (NN) as a powerful learning tool may allow improving the existing model to learn from previous knowledge and potentially to utilise it in knowledge modelling and acquisition.

Keywords: Supplier Evaluation \& Selection, Simulation, MATLAB® \& SIMULINK®, Multi-Criteria Decision System 


\section{INTRODUCTION}

Supplier evaluation and selection (SE\&S) is perhaps one of the most important functions of the purchasing department in a supply chain. The selection of the "best" supplier has huge impact on both short and long term performance of the organisation and the supply chain as a whole. The selection process is a complicated one as it requires collecting accurate information as an input to the decision model, selecting a suitable model to process that information and then selecting the best supplier or a list of the best possible suppliers. Adding to the complexity of the problem, in many real life situations, data available for the decision makers is usually uncertain, sometimes vague or incomplete. The task of establishing a robust, practical and accurate model to represent the problem and provide the most accurate solution is still far from accomplished(Liao and Rittscher 2005, Abu-Ajamieh, Luong et al. 2009).

The main objective of this paper is to develop a simulation model utilising the powerful and flexible resources in MATLAB® and SIMULINK® to represent and solve the SE\&S problem in a way that takes into consideration the complexity of the problem and the variety of selection criteria.

\section{LITERATURE REVIEW}

Supplier selection problem can be defined as how to evaluate and select the best supplier from a population of suppliers, given a set of conflicting criteria (such as quality, delivery, price, capacity, etc.). Literature is full of researches and models that attempted to model and solve this problem under different conditions, constraints and assumptions and using a multitude of techniques and methodologies. It is not the purpose of this paper to cover all those models; however it is essential to review some of the most important ones. In the following section, a brief review of supplier selection methods is presented.

\subsection{Review of supplier selection methods}

Supplier selection methods as seen in the literature can be categorised in many different ways. In this review, we choose to split them into two main categories; Traditional and non-traditional methods.

\section{Traditional Methodologies}

Traditionally, the supplier selection problem was modelled using many different techniques. The majority of them are based on the assumption of quantitative inputs and the use of deterministic methods. Some of the most common methodologies found in the literature are listed below:

1. Mathematical Programming

2. Statistical methods

3. Linear weighing methods

4. Total Cost of Ownership

5. Interpretative Structural modelling (ISM)

6. Analytical Hierarchy Process (AHP)

7. Data Envelopment Analysis (DEA)

8. Hybrid systems that combine more than one of the traditional methods.

9. Simulation Models

The multi-objective nature of the supplier selection problem was analysed by Weber in 1993 (Weber and Current 1993) to formulate the selection problem as multi-objective integer programming problem and then apply it to a specific case study. Thus this model was limited to a certain specific situation, and in its best generalised shape will be a very complex mathematical model which may be impractical for use or hard to solve(Liao and Rittscher 2005, Abu-Ajamieh, Luong et al. 2009). Other researchers also attempted to model the supplier selection decision problem using other tools and techniques. For example, Interpretive Structural modelling (ISM) was introduced as a new method to supplier selection (Mandal and Deshmukh 1994). In their study they chose only 11 criteria out of the 23 criteria documented in the literature taking into consideration the Indian engineering industry. 
Ghodsypour \& O'Brien (2001) used a mixed integer non-linear programming to model and solve the problem of supplier selection taking into consideration new factors such as transportation costs, ordering and storage costs which may be important to the decision makers.

Hybrid models have been also used to solve the supplier selection problem in order to improve accuracy and performance of the decision model and to be able to deal with the increased number of criteria (Weber, Current et al. 1998) used multi objective programming $\&$ data envelopment analysis for vendor selection in a specific application in an environment of competing vendors where some vendors are not selected and there is a need to analyse their performance in order to make them more competitive.

However, the most commonly used technique in the supplier selection problem was the Analytical Hierarchy Process (AHP), used separately or combined with other techniques for better results. For example AHP and linear programming were used to analyse both qualitative and quantitative data (Ghodsypour and O'Brien 1998). In their work, they introduced the level of buyer-supplier relationship as an important factor for deciding the number of criteria to be selected.

An important development in this area was the use of expert systems as a tool for supplier evaluation and selection (Vokurka, Choobineh et al. 1996), where they modelled the evaluation and selection decision process using the expert's knowledge extracted from purchasing manager of a process manufacturing company.

The common factor between all traditional techniques and the models built based on them is that they tend to model problems with limited number of selection criteria. In addition, they consider only quantitative type of data, which is not the case in practical situation.

\section{Non Traditional Methodologies}

The non traditional models presented here are mainly those which are based Fuzzy Logic and Neural Networks or combine with one of the other traditional models.

One of the emerging methodologies in the supplier selection problem was the implementation of the Fuzzy Set Theory and Fuzzy Logic. (Bevilacqua and Petroni 2002). The researchers have investigated the previous supplier selection methodologies and came to conclusion that due to uncertainties in the problem they used fuzzy-based approach to represent the model. The model was based on building a Fuzzy suitability index (FSI) as a method of ranking the suppliers. The selection criteria were identified using a panel of experts reviewing a wide range of criteria identified in the literature. The growing interest in using fuzzy logic and fuzzy systems in the supplier selection area can easily be noticed from the increased number of researches performed in the last few years.(Bevilacqua and Petroni 2002, Chang, Wang et al. 2006, Bayrak, C?elebi et al. 2007)

Another development in the supplier selection problem was the use neural networks (NN). This was proposed by Albino and Garavelli in their study of subcontractor rating in construction companies (Albino and Garavelli 1998). The model was based on teaching a NN by examples. The use of NN gave their model some important strength; such as simplifying the decision making process since there is no need to be stuck in detailed algorithms to apply the model, nevertheless, it has its inherent limitations if used alone. The model did not include uncertainty which is a very important factor in real life situation.

The aim of this paper is to provide a new approach to representing and solving the SE\&S decision problem with multiple number of selection criteria of different types taking into account uncertainty and vagueness, using simulation in order to build a model that is more representative of the human decision making process.

\section{MODEL DESCRIPTION:}

The methodology is based on the concept of Supplier Product Profile (SPP) and its Equivalent cost (SPPEC), Abu-Ajamieh, Luong et al. (2009). 


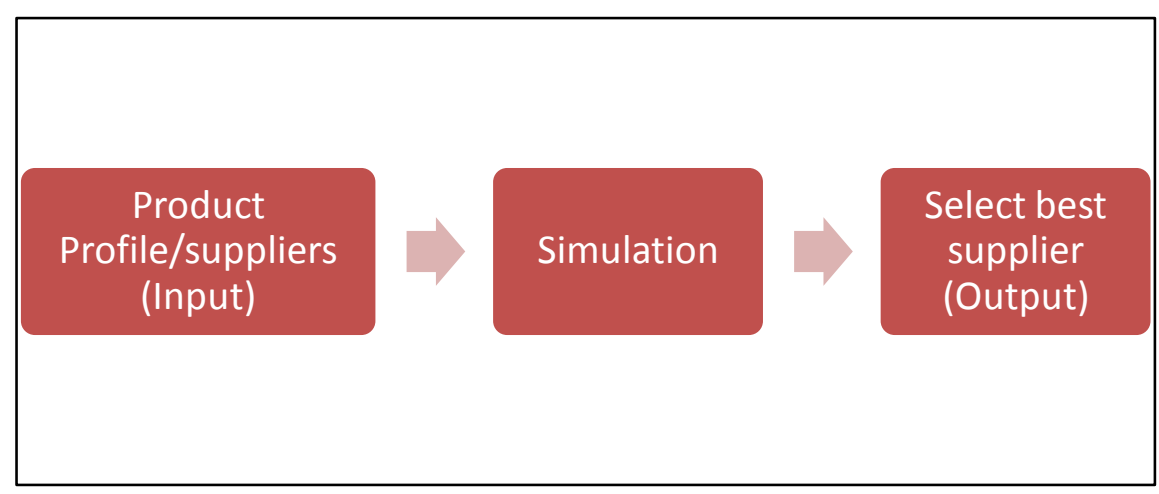

Figure1. Schematic diagram of the Model

To build a SES decision model, we need to consider the types of input data (decision criteria) to the model. Decision criteria of the supplier SE\&S model are either related to the product/component itself such as physical attributes, dimensions, shape, etc or the supplier production and management system related attributes such as quality certification, process quality, delivery performance.etc or actually that is related to both supplied product and supplier system performance such as component price, capability index, etc.

Those attributes/criteria are not fixed, but rather dynamic in nature because of the natural variability in the processes, production and management systems, market, competition and so on, so the values of these performance measures/attributes will change over time, but may follow certain behaviour, rules or functions that may be described by a distribution, specification limits, parts per million (PPM), etc which are the measures used by industry to describe product/process behaviour.

Also, these criteria are of different types in nature, and what we mean by type here is the method of describing, measuring, or evaluating these criteria. For example; we may have quantitative values such as length in $\mathrm{mm}$, the process capability index (Cpk) or delivery performance as percentage of on time delivery. On the other hand, some criteria- and many times in real life situation it is the case- are qualitative or descriptive, for example, acceptable price, good quality, average service and so on. The scheme for treating such variations in selection criteria data types was based on the concepts presented in (Abu-Ajamieh, Luong et al. 2009)

In many cases, the criteria may have some uncertainties that can be quantified using statistical means i.e. confidence intervals. All these variations are some of challenges that face the decision maker and the modeller in terms of how to deal with them, evaluate them properly or include them in the decision making process.

To build a model using a specific real life case study, it might be expected that such model will not be general, but rather specific to that case study and its limitations in terms of number of suppliers, numbers of evaluation criteria used in the case/company, the type of data measures used and soon. So this methodology will not be good enough to build a robust, generalised model.

For all the above, the methodology proposed here is an attempt to tackle some of these problems by using a scenario based simulation model which is based on a specifically designed case study that includes all these variations and complexities in the problem in order to demonstrate the strength of this solution approach.

In this model and for the sake of simplicity and demonstration only we are investigating the supply of a product by four different suppliers by evaluating their performance in three selection criteria (quality, delivery and price) where:

- Each supplier system has the same number/type of performance measures (this assumption is not necessary needed for the generic model but to simplify the demonstration only, any type of performance /decision criteria can be dealt with). It is assumed that data is available.

- The decision makers have clearly specified their preferred or ideal performance for each criterion. This is called the Ideal Supplier Product profile (ISPP) and will be fixed for a given situation/case/product. 
For example, management may decide that the preferred price for component "a" is $\$ 10$, and then this will be considered for all suppliers providing this component.

- The management will also clearly identify any penalty measures for any criteria type in case of deviation from the ideal/preferred situation. This is critically needed in order to convert all criteria into one measure which is the dollar value we call it the supplier product equivalent cost (SPPEC).

- In addition, each criteria/sub criteria need to have a coefficient of importance as some criteria may be more important to meet than others and hence will have more weight. This will help management reach a more accurate and representative decision of what they want.

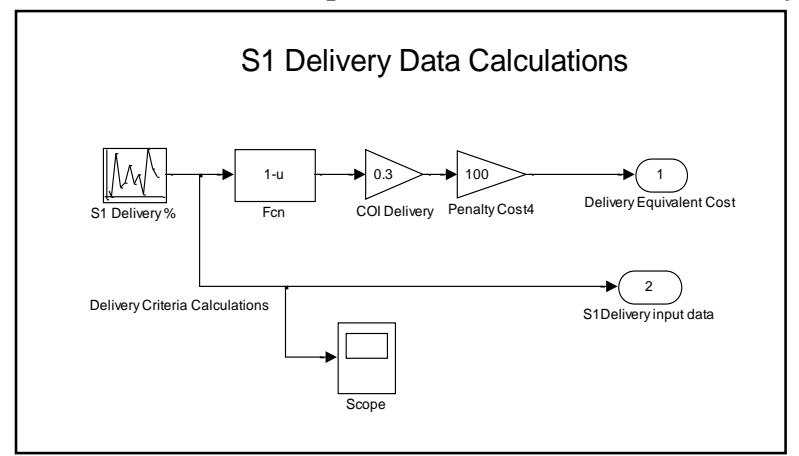

Figure 2. Simulating Supplier 1 Delivery Performance

In the simulation model, all suppliers will generate products based on a known distribution or quantity, in addition, the decision attributes of the component/supplier will also be generated based on the supplier own unique process and management system description.

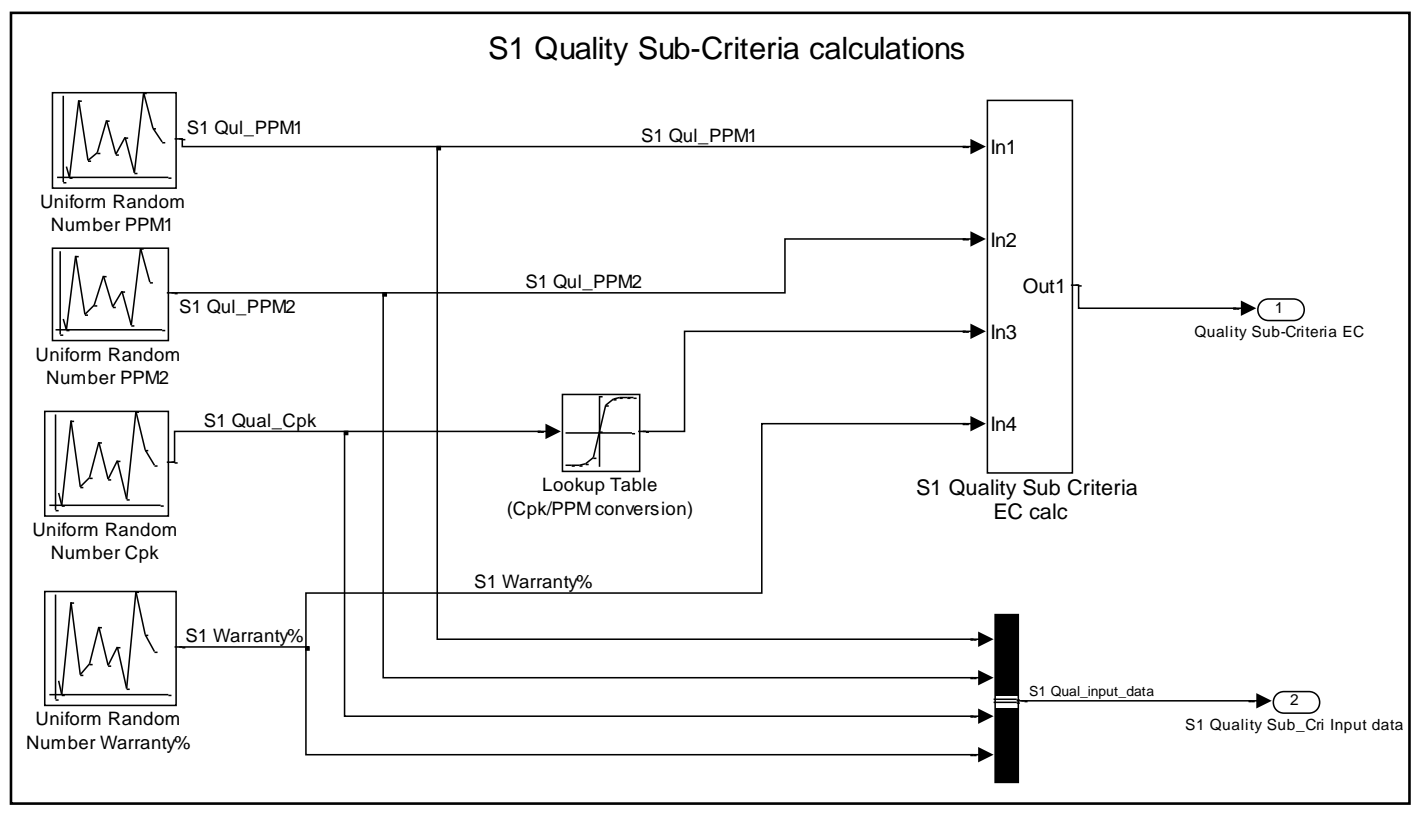

Figure 3. Simulating Supplier 1 Quality Performance

Utilising the integrated environment of MATLAB® and SIMULINK® and the available toolboxes such as Fuzzy Logic tool box, any type of input criteria/performance behaviour can be modelled as long as the characteristics of the process/product are specified, for example: for supplier1, the product and supplier attributes (performance) is described by 3 major criteria of different types (quality, delivery and price), quality has four sub-criteria, each criteria/performance is described using a SIMULINK® function with specific parameters that represent the unique situation of that supplier/component: Product quality PPM described a quality sub criteria which evaluates part per million defects of the product quality characteristic this is characterised by a uniformly distributed random number of minimum 45 and a maximum of 55 and so forth. 
All performance attributes are fed into a block that calculates the actual supplier-product equivalent cost (ASPPEC) and compares is with the ISPPEC for each product, supplier, in every simulation run/iteration. This will generate a range of supplier performances over time that mimics the behaviour of the supplier and product performances in the real life situation.

The supplier that generates the best performance in all attributes and over the overall simulation run (studied life span) will be selected. This is done by selecting the supplier that has minimum ASPPEC (averaged over the simulation runs). This way, we can mimic any production run or time span, any kind of supplier performance or product characteristics using simulation and then it will be easier to select best supplier based on a measurable and easy to interpret dollar value. In real life, instead of using simulated inputs, this model can accept the real data, convert them into ASPPEC and compare them with the ISPPEC and select the best supplier. All that is needed would be to specify from the beginning, what decision criteria are considered, needed, or available, their importance and costs/penalties associated with them not meeting their preferred levels.

\section{SIMULATION RESULTS}

The simulated performances of all four suppliers (for 100 simulation runs) are shown in Figure 5. We can clearly see that supplier 4 is the one with the minimum equivalent cost for the whole simulation. Taking into consideration the performance of supplier over all different selection criteria and averaging it over the simulation runs. This supplier would be selected as the best supplier for the long term. This way the best supplier is selected not based on one single performance but rather on its performance over a desired period of time ( as decided by managers) and across all required selection criteria, thus the decision mad is expected to be more accurate and reliable.

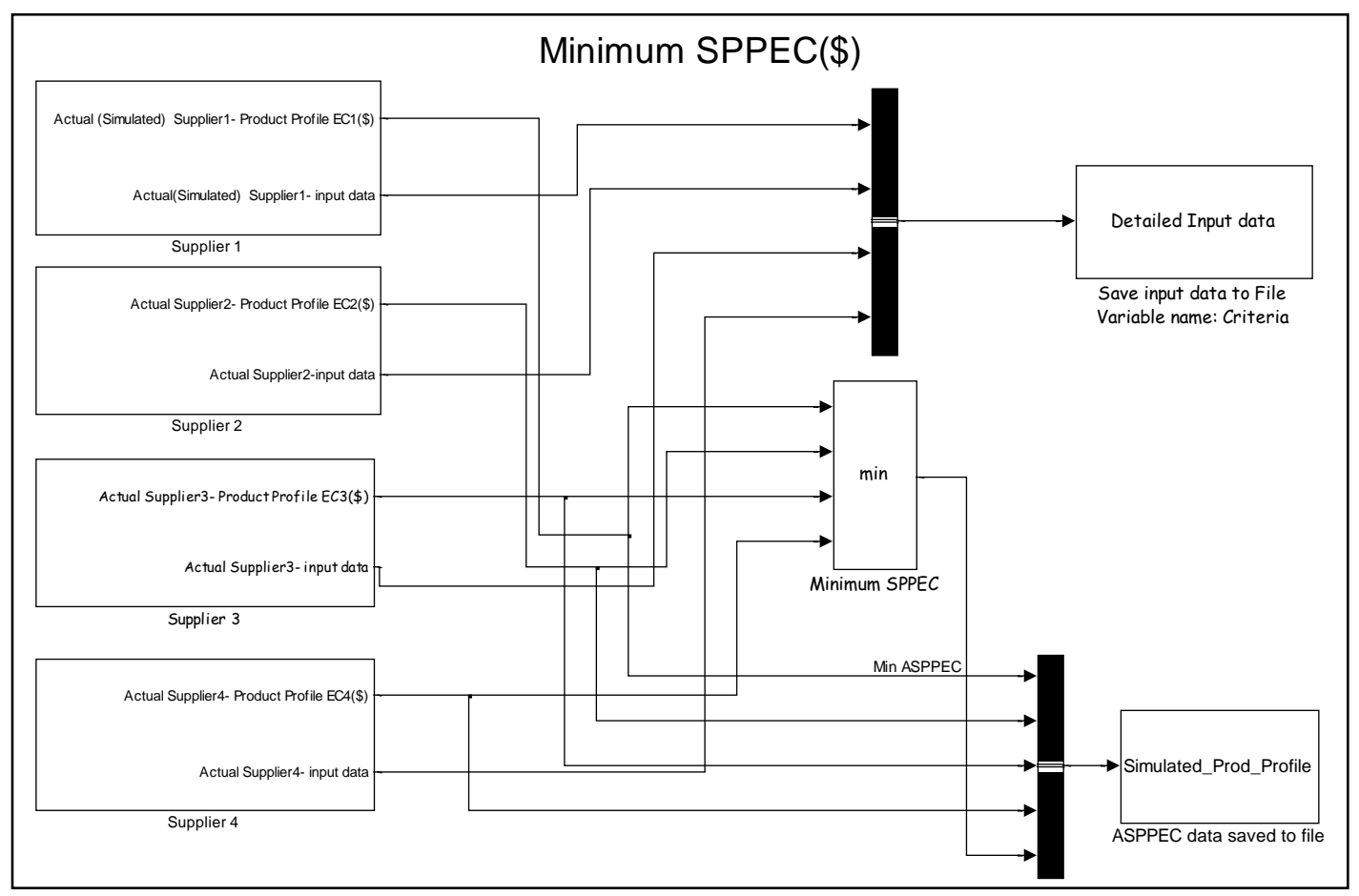

Figure 4. Selecting the best supplier using Minimum Equivalent Cost

\section{DISCUSSION AND CONCLUSION/FURTHER RESEARCH:}

A new SIMULINK® Simulation model has been presented here as an extension to previously published SE\&S model(Abu-Ajamieh, Luong et al. 2009) to assist the decision makers in the evaluation and selection of new and current suppliers. The model utilises the strengths of simulation to establish a more robust tool that takes into consideration changes in supplier performances over time across all selection criteria. It can be clearly noticed that it is a simple model and easy to use. 


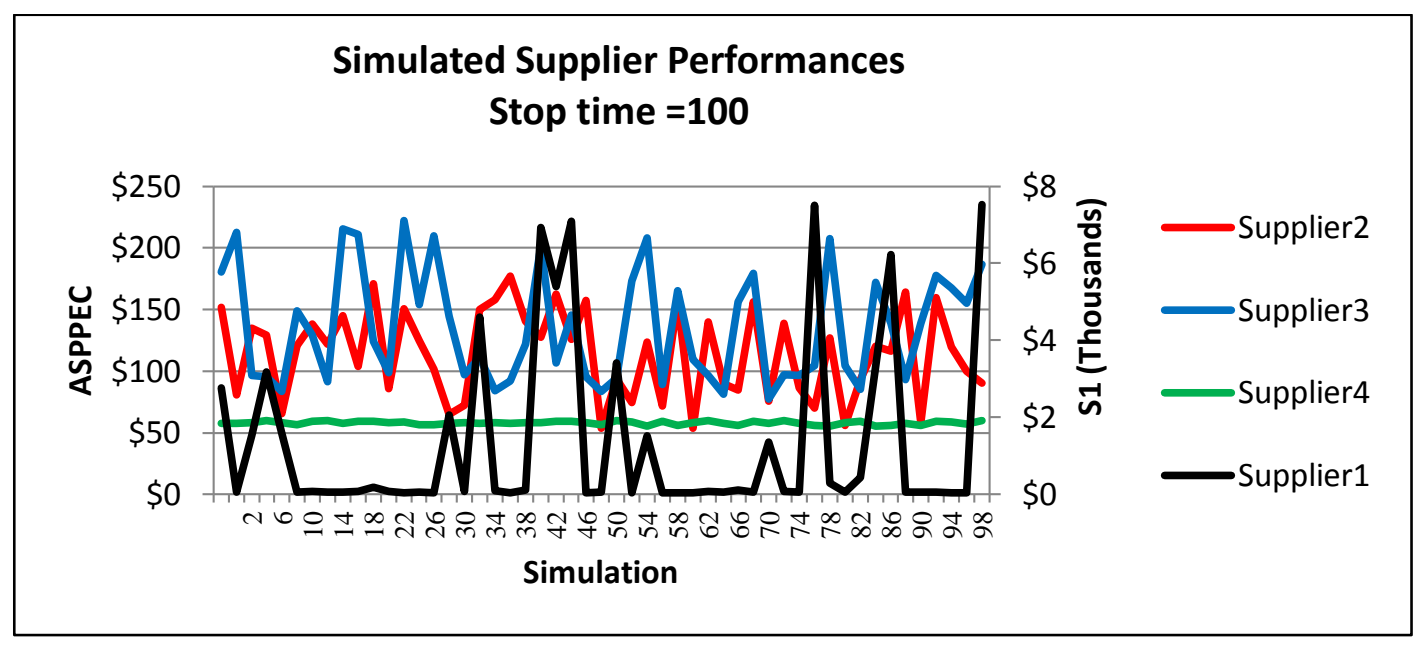

Figure 5. Simulation Results for four Suppliers

The decision model has two distinctive advantages over other models in terms of capability to model and solve SE\&S problems with unlimited number of selection criteria, potential suppliers and any type of input data (qualitative or quantitative). The only constraints that can be identified in this model may come from the simulation software limitation on number and sizes of input variables. Another important advantage of this model would stems from its flexibility and wide range of potential future applications as it is very easy to utilise the concept to model and solve any multi-alternative decision problem. Future work suggested for this model would be to investigate using Neural Networks (NN) to allow the model to learn from previous knowledge and potentially to utilise the model in knowledge modelling and acquisition.

\section{REFERENCES}

Abu-Ajamieh, A. M., L. Luong and R. Marian (2009). SUPPLIER EVALUATION \& SELECTION USING SUPPLIER-PRODUCT PROFILING 7th ANZAM Operations, Supply Chain and Services Management Symposium 2009, Adelaide.

Albino, V. and C. Garavelli (1998). "A neural network application to subcontractor rating in construction firms." International journal of Project Management 16(1): 9-14.

Bayrak, M. Y., N. C?elebi and H. Takin (2007). "A fuzzy approach method for supplier selection." Production Planning and Control 18(1): 54-63.

Bevilacqua, M. and A. Petroni (2002). "From Traditional Purchasing to Supplier Management: A Fuzzy Logicbased Approach to Supplier Selection." International Journal of Logistics Research and Applications 5(3): 235 255.

Chang, S. L., R. C. Wang and S. Y. Wang (2006). "Applying fuzzy linguistic quantifier to select supply chain partners at different phases of product life cycle." International Journal of Production Economics 100(2): 348359.

Ghodsypour, S. H. and C. O'Brien (1998). "A decision support system for supplier selection using an integrated analytic hierarchy process and linear programming." International Journal of Production Economics 56-57: 199212.

Liao, Z. and J. Rittscher (2005). "A multi-objectice supplier selection model under stochastic demand conditions." International journal of production economics 105: 150-159.

Mandal, A. and S. G. Deshmukh (1994). "Vendor Selection Using Interpretive Structural Modelling (ISM)." International Journal of Operations \& Production management 14(6): 52-59.

Vokurka, R. J., J. Choobineh and L. Vadi (1996). "A prototype expert system for the evaluation and selection of potential suppliers." International Journal of Operations \& Production management 16(12): 106-127.

Weber, C. A. and J. R. Current (1993). "A multiobjective approach to vendor selection." European Journal of Operational Research 68(2): 173-184.

Weber, C. A., J. R. Current and A. Desai (1998). "Non-cooperative negotiation strategies for vendor selection." European Journal of Operational Research 108(1): 208-223. 